\title{
The Qualitative Analysis for a Class Nonlinear Systems with High Degree
}

\author{
Songlin Chen*, Yajie Du \\ School of Mathematics \& Physics, Anhui University of Technology, Ma’anshan, China \\ Email: *slchen@ahut.edu.cn
}

How to cite this paper: Chen, S.L. and Du, Y.J. (2018) The Qualitative Analysis for a Class Nonlinear Systems with High Degree. Journal of Applied Mathematics and Physics, 6, 1006-1011.

https://doi.org/10.4236/jamp.2018.65087

Received: June 16, 2017

Accepted: May 19, 2018

Published: May 22, 2018

Copyright $\odot 2018$ by authors and Scientific Research Publishing Inc. This work is licensed under the Creative Commons Attribution International License (CC BY 4.0).

http://creativecommons.org/licenses/by/4.0/

\begin{abstract}
In the paper, the problems of identification of focus or center for a class of nonlinear systems are studied. As results, the identifying rule for the type of singular point of the system and the calculating formula for the focus value are obtained by the classical theory of Poincare and Lyapunov. At the end, the numerical simulation for a polynomial system of seven-degree are showed to corroborate the theoretical results of the method.
\end{abstract}

\section{Keywords}

Focus, Center, Focus Quantities, Polynomial System

\section{Introduction}

A class of plane polynomial nonlinear systems with a singular point are studied in the paper. We know that the singular point maybe a center or a focus, thus the identification for focus or center need to be performed. The problem of identification for focus and center has inseparable relationship to the study of stability of the system. The conventional methods are the methods of successive function and the formal series [1] [2] [3]. This kind of systems arise frequently in many applied science fields such as biomathematics, fluid dynamics, quantum mechanics, chemical reaction and so on [4] [5]. In the paper, the center or focus of a class of nonlinear system with higher degree polynomial are analyzed by using the methods in [6] [7] [8].

The paper is organized in the following style: in Section 2, the identification method is constructed, and the calculating formula for the focus value is obtained. At the end, in Section 3, a seven-degree polynomial system is numerically simulated. 


\section{Main Results}

The following nonlinear system with higher degree polynomial

$$
\left\{\begin{array}{l}
\dot{x}=-2 x^{2} y-y^{3}+x^{3} y^{2} f(x, y) \\
\dot{y}=x^{3}+x^{2} y^{3} f(x, y)
\end{array}, \text { of }(x, y) \geq 1\right.
$$

are considered.

Conventionally, applying the polar coordinates

$$
\left\{\begin{array}{l}
x=r \cos \theta \\
y=r \sin \theta
\end{array}\right.
$$

to the System (1) results in

$$
\begin{aligned}
\frac{\mathrm{d} r}{\mathrm{~d} \theta} & =-r * \cos \theta * \sin \theta+r^{3} * \cos ^{2} \theta * \sin ^{2} \theta * f(r \cos \theta, r \sin \theta): \\
& =R(r, \theta)
\end{aligned}
$$

Express initial value problem of the differential Equation (2) as following form

$$
\left\{\begin{array}{l}
\frac{\mathrm{d} r}{\mathrm{~d} \theta}=R(\theta, r)=\sum_{i=1}^{\infty} R_{i}(\theta) r^{i}=R_{1}(\theta) r+R_{2}(\theta) r^{2}+\cdots+R_{k}(\theta) r^{k}+\cdots \\
r(0)=c=\text { constant }
\end{array}\right.
$$

Moreover, assume the formal series solution for problem (3) is

$$
r(\theta, c)=\sum_{i=1}^{\infty} r_{i}(\theta) c^{i}=r_{1}(\theta) c+r_{2}(\theta) c^{2}+\cdots+r_{j}(\theta) c^{j}+\cdots
$$

Substitute (4) into (3) gives

$$
\sum_{i=1}^{\infty} r_{i}^{\prime}(\theta) c^{i}=\sum_{k=1}^{\infty}\left[R_{k}(\theta)\left(\sum_{i=1}^{\infty} r_{i}(\theta) c^{i}\right)^{k}\right]
$$

Expand the right hand of the equality and compare the bilateral coefficients of $c^{i}$, the results are stated as follows

$$
\begin{gathered}
\left\{\begin{array}{l}
\frac{\mathrm{d} r_{1}}{\mathrm{~d} \theta}=R_{1}(\theta) * r_{1}(\theta) \\
r_{1}(0)=1
\end{array}\right. \\
\left\{\begin{array}{l}
\frac{\mathrm{d} r_{k}}{\mathrm{~d} \theta}=R_{1}(\theta) * r_{k}(\theta)+Q_{k}\left(R_{1}, R_{2}, \cdots, R_{k} ; r_{1}, r_{2}, \cdots, r_{k-1}\right), k \geq 2 \\
r_{k}(0)=0
\end{array}\right.
\end{gathered}
$$

thus $r_{1}(\theta), r_{2}(\theta), \cdots, r_{k}(\theta), \cdots$ are solved successively and the series solution of the problem (3) is thus obtained by (4).

Next we'll obtain an equivalent description to the focus value $r(2 \pi, c)$.

Denote the first integral of (3) by

$$
c=\varphi(\theta, r)=\sum_{i=1}^{\infty} \varphi_{i}(\theta) r^{i}
$$

then we have the following results.

Theorem System (1) or (2) has the following recurrence formula: 


$$
\begin{aligned}
& 1^{\circ}\left\{\begin{array}{l}
\varphi_{1}^{\prime}(\theta)=-R_{1}(\theta) * \varphi_{1}(\theta) \\
\varphi_{n}^{\prime}(\theta)=-\sum_{k=1}^{n} k * R_{n-k+1} * \varphi_{k}(\theta), n \geq 2 \\
\varphi_{1}(0)=1, \varphi_{k}(0)=0, k \geq 2
\end{array}\right. \\
& 2^{\circ} \varphi_{n}(2 \pi)=-r_{n}(2 \pi) \forall n \geq 2, n \in N
\end{aligned}
$$

Proof Because

$$
c=\varphi(\theta, r)=\sum_{i=1}^{\infty} \varphi_{i}(\theta) r^{i}=r(0)
$$

we have

$$
\frac{\partial \varphi}{\partial \theta}+R(\theta, r) \frac{\partial \varphi}{\partial r}=0
$$

substitute $\varphi(\theta, r)=\sum_{i=1}^{\infty} \varphi_{i}(\theta) r^{i}$ and $R(\theta, r)=\sum_{i=1}^{\infty} R_{i}(\theta) r^{i}$ into the partial differential equation, and expand the equality as follows

$$
\begin{aligned}
& {\left[\varphi_{1}^{\prime}(\theta) r+\varphi_{2}^{\prime}(\theta) r^{2}+\varphi_{3}^{\prime}(\theta) r^{3}+\varphi_{4}^{\prime}(\theta) r^{4}+\cdots+\varphi_{m}^{\prime}(\theta) r^{m}+\cdots\right]} \\
& +\left[R_{1}(\theta) \varphi_{1}(\theta) r+2 R_{1}(\theta) \varphi_{2}(\theta) r^{2}+3 R_{1}(\theta) \varphi_{3}(\theta) r^{3}+4 R_{1}(\theta) \varphi_{4}(\theta) r^{4}\right. \\
& \left.+2 R_{1}(\theta) \varphi_{5}(\theta) r^{5}+\cdots+m R_{1}(\theta) \varphi_{m}(\theta) r^{m}+\cdots\right] \\
& +\left[R_{2}(\theta) \varphi_{1}(\theta) r^{2}+2 R_{2}(\theta) \varphi_{2}(\theta) r^{3}+3 R_{2}(\theta) \varphi_{3}(\theta) r^{4}+4 R_{2}(\theta) \varphi_{4}(\theta) r^{5}\right. \\
& \left.+5 R_{2}(\theta) \varphi_{5}(\theta) r^{6}+\ldots+m R_{2}(\theta) \varphi_{m}(\theta) r^{m+1}+\cdots\right] \\
& +\left[R_{3}(\theta) \varphi_{1}(\theta) r^{3}+2 R_{3}(\theta) \varphi_{2}(\theta) r^{4}+3 R_{3}(\theta) \varphi_{3}(\theta) r^{5}+4 R_{3}(\theta) \varphi_{4}(\theta) r^{6}\right. \\
& \left.+5 R_{3}(\theta) \varphi_{5}(\theta) r^{7}+\cdots+m R_{3}(\theta) \varphi_{m}(\theta) r^{m+2}+\cdots\right] \\
& +\cdots=0
\end{aligned}
$$

Comparing the bilateral coefficients of $r^{i}$ gives the conclusion $1^{\circ}$ directly.

To prove $2^{\circ}$, with the expressions $c=\varphi(\theta, r)=\sum_{i=1}^{\infty} \varphi_{i}(\theta) r^{i}=r(0)$ and (4), we have $r=\sum_{j=1}^{\infty} r_{j}\left(\sum_{i=1}^{\infty} \varphi_{i} r^{i}\right)^{j}$, that is

$$
\begin{aligned}
r= & r_{1}\left[\varphi_{1} r+\varphi_{2} r^{2}+\cdots\right]+r_{2}\left[\varphi_{1} r+\varphi_{2} r^{2}+\cdots\right]^{2}+r_{3}\left[\varphi_{1} r+\varphi_{2} r^{2}+\cdots\right]^{3} \\
& +\cdots+r_{m}\left[\varphi_{1} r+\varphi_{2} r^{2}+\cdots\right]^{m}+\cdots
\end{aligned}
$$

From the identity it is obvious that

$$
\varphi_{2}(2 \pi)=-r_{2}(2 \pi), \quad \varphi_{3}(2 \pi)=-r_{3}(2 \pi)
$$

with the help of the method of mathematical induction, $\varphi_{n}(2 \pi)=-r_{n}(2 \pi)$ $\forall n \geq 2, n \in N$ will be true.

According to the classical theory of Poincaré and Lyapunov, it is well known that when $r_{k}(2 \pi, c)$ is non-zero, its subscript must be an odd number. If $r_{2 k+1}(2 \pi, c)=0$ for all $k$, then the original point is a center. If $r_{2 k+1}(2 \pi, c) \neq 0$ for some specified $k$, then the original point is a $k$-order fine focus; meanwhile, 
$r_{k+1}(2 \pi, c)$ is the focus value of the $k$-order fine focus, according to the theorem above we known $\varphi_{2 k+1}(2 \pi)$ can be regarded as an equivalent description for the $k$-order fine focus.

\section{Simulations}

Consider the specified systems:

$$
\left\{\begin{array}{l}
\dot{x}=-2 x^{2} y-y^{3}+x^{3} y^{2} f(x, y) \\
\dot{y}=x^{3}+x^{2} y^{3} f(x, y)
\end{array}, \partial f(x, y) \geq 1\right.
$$

wherein $f(x, y)=a_{1} x+b_{1} y+a_{2} x^{2}+b_{2} y^{2}$. The system can be expressed by polar coordinate $(r, \theta)$ such as

$$
\begin{aligned}
\frac{\mathrm{d} r}{\mathrm{~d} \theta}= & -r * \cos \theta * \sin \theta+r^{3} * \cos ^{2} \theta^{*} \sin ^{2} \theta * f(r \cos \theta, r \sin \theta) \\
:= & R(r, \theta)=-r \sin \theta \cos \theta+r^{4}\left(a_{1} \cos ^{3} \theta \sin ^{2} \theta+b_{1} \cos ^{2} \theta \sin ^{3} \theta\right) \\
& +r^{5}\left(a_{2} \cos ^{4} \theta \sin ^{2} \theta+b_{2} \cos ^{2} \theta \sin ^{4} \theta\right)
\end{aligned}
$$

thus the following relationships are obtained

$$
\begin{gathered}
R_{1}(\theta)=-\sin \theta \cos \theta, R_{2}(\theta)=0, R_{3}(\theta)=0 \\
R_{4}(\theta)=a_{1} \cos ^{3} \theta \sin ^{2} \theta+b_{1} \cos ^{2} \theta \sin ^{3} \theta \\
R_{5}(\theta)=a_{2} \cos ^{4} \theta \sin ^{2} \theta+b_{2} \cos ^{2} \theta \sin ^{4} \theta
\end{gathered}
$$

The recurrence formula which quoted from above is stated as follows

$$
\left\{\begin{array}{l}
\varphi_{1}^{\prime}(\theta)=-R_{1}(\theta) * \varphi_{1}(\theta) \\
\varphi_{n}^{\prime}(\theta)=-\sum_{k=1}^{n} k * R_{n-k+1} * \varphi_{k}(\theta) \quad n \geq 2 \\
\varphi_{1}(0)=1, \varphi_{k}(0)=0 \quad k \geq 2
\end{array}\right.
$$

Moreover, for simulating the result, we specify the parameters in system involved in $f(x, y)$ as

$$
a_{1}=1, b_{1}=2, a_{2}=-3, b_{2}=4
$$

According to Theorem in Section 2, it is sufficient to investigate the values of $\varphi_{2 k+1}(2 \pi)$ which can be obtained approximately by the drawings of $\varphi_{3}(\theta)$ and $\varphi_{5}(\theta)$ on interval $[0,2 \pi]$ as follows:

Figure 1, Figure 2 and the numerical result show that $\varphi_{3}(2 \pi)=0$ and $\varphi_{5}(2 \pi)=0.0784437 \neq 0$, thus according to the Theorem in Section 2, we know that the singular point of system $(10-11),(0,0)$, is a 2 -order fine focus with the focus value being -0.0784437 .

To verify the results above, the phase portrait of the specified system (10-11) is depicted directly by Mathematica as in Figure 3.

Figure 3 shows that the original point $(0,0)$ is a focus which corroborates in practice the theoretical analysis of the method in the paper. 


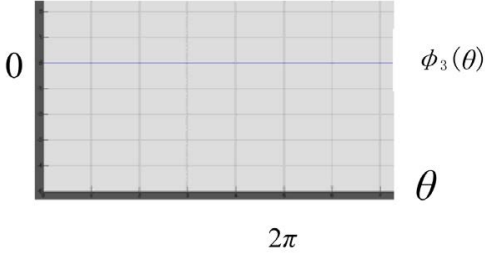

Figure 1. $\varphi_{3}(\theta)$.

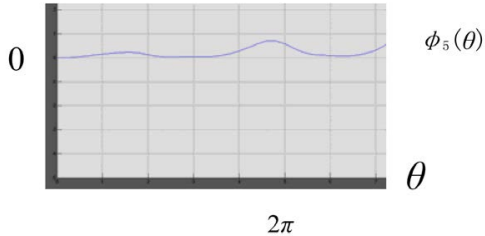

Figure 2. $\varphi_{5}(\theta)$.

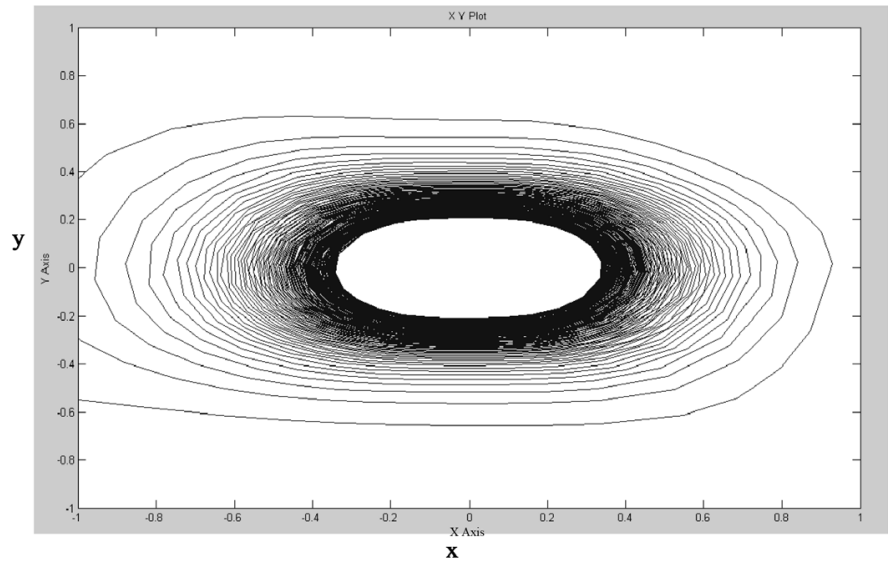

Figure 3. The phase portrait of the specified system (10), (11).

\section{Conclusion}

The paper considers the problems of identification of focus or center for a class of nonlinear systems. As results, the identifying rule for the type of singular point of the system and the calculating formula for the focus value are obtained by the classical theory of Poincare and Lyapunov. The numerical simulation shows that the theoretical results of the method is valid.

\section{Acknowledgements}

Authors express their grateful to the reviewer for giving comments on the revision of the paper.

\section{Funding}

The research supported by the National Natural Science Foundation of China (Grant No. 50975003) and The Major Project of Natural Science from Education Department of Anhui Province (KJ2016A084). 


\section{References}

[1] Zhang, Z. and Ding, T. (1986) The Qualitative Theory of the Differential Equation. Science Press, Beijing.

[2] Luo, J., Zhang, X. and Dong, M. (2001) The Qualitative and Bifurcation Theory of the Dynamics System. Science Press, Beijing.

[3] Han, M. and Gu, S. (2001) The Theory and Method of the Nonlinear System. Science Press, Beijing.

[4] Du, N. and Zeng, X. (1994) A Class of Recurrence Formula for Calculating Focus Value. Science Bulletin, 39, 1742-1744.

[5] Yue, X. (2005) The Identification of Center and Focus for a Class of Poincaré Equation. Advances in Mathematics, 34, 101-105.

[6] Ren, Q.J., Huang, Y.K. and Li, F. (2012) Computing Center Conditions for Non-Analytic Ninth Degree Vector Fields with Constant Angular Speed. Advances in Difference Equations, 2012, 231. https://doi.org/10.1186/1687-1847-2012-231

[7] Wang, Q.L. and Huang, W.T. (2012) The Equivalence between Singular Point Quantities and Liapunov Constants on Center Manifold. Advances in Difference Equations, 2012, 78. https://doi.org/10.1186/1687-1847-2012-78

[8] Chen, S.L. and Nian, Y.D. (2007) The Recursive Calculation of Higher Order Focus Values for a Class of Nonlinear Systems. Acta Physica Sinica, 56, 1851-1854. 